\title{
Activation of the nitric oxide-cGMP signaling pathway precedes resolution in acute anti-thy I glomerulonephritis
} Yingrui Wang-Rosenke*, Stephanie Krämer, Tanja Loof, Sebastian Martini, Dmytro Khadzhynov, Eva Binder, Hans-Hellmut Neumayer and Harm Peters

\author{
Address: Charité Universitätsmedizin Berlin, Charité Campus Mitte, Department of Nephrology, Berlin, Germany \\ Email: Yingrui Wang-Rosenke* - yingrui.wang@charite.de \\ * Corresponding author
}

 \\ Dresden, Germany. I5-17 June 2007 \\ Published: 25 July 2007 \\ BMC Pharmacology 2007, 7(Suppl I):P63 doi:I0.II86/I47|-22I0-7-SI-P63
}

This abstract is available from: http://www.biomedcentral.com/I47I-22 I0/7/SI/P63

(c) 2007 Wang-Rosenke et al; licensee BioMed Central Ltd.

\begin{abstract}
Aim
Resolution of acute anti-thy1 glomerulonephritis by itself indicates an endogenous program of disease regression is operating. Given the renoprotective effects of the endothelial nitric oxide (NO)-cGMP pathway, the present study analyzed expression and activity of NO-cGMP signaling during a time course of acute anti-thy1 glomerulonephritis.
\end{abstract}

\section{Methods}

Rats with anti-thy1 glomerulonephritis were sacrificed at 0.5 and 1 day (injury), 5 and 10 days (matrix expansion) and 15 and 20 days (resolution) after OX-7 antibody injection. Measures of disease activity included glomerular matrix accumulation, transforming growth factorbeta1 (TGF-beta1) expression as well as glomerular platelet deposition, macrophage infiltration and cell proliferation. NO-cGMP signaling was characterized by measuring glomerular NO and cGMP production as well as glomerular mRNA expression of endothelial NO synthase (eNOS), inducible NOS (iNOS), alpha1 and beta1 soluble guanylate cyclase (sGC) and phosphodiesterase-5 (PDE-5). Results are expressed in relation to normal day 0 controls $(=100 \%)\left({ }^{*} \mathrm{p}<0.05\right.$ versus normal day 0 controls $)$.

\section{Results}

In the injury phase, mesangial cell lysis was paralleled by markedly induced glomerular NO production $(+673 \% *$, iNOS expression $+742 \% \%^{*}$ ), but loss of NO-stimulated glomerular cGMP production $(-63 \% *)$ due to reduced alpha1 $\left(-63 \% \%^{*}\right)$ and beta1 sGC expression $\left(-63 \% \%^{*}\right)$. Disruption of NO-cGMP signaling was followed by marked increases in glomerular TGF-beta 1 expression $\left(+545 \%{ }^{*}\right)$, matrix accumulation $(+187 \% *)$, platelet deposition $\left(+5677 \% \%^{*}\right)$, leukocyte infiltration $\left(+513 \%{ }^{*}\right)$ and cell proliferation $\left(+432 \%^{*}\right)$ at day 5 (matrix expansion phase). Glomerular disease indicators returned towards normal at day 20. Resolution of glomerular matrix expansion was preceded by a significant up-regulation of NO-stimulated glomerular cGMP $(+8860 \% *)$, alpha1 $(+1459 \% *)$ and beta1 sGC $\left(+972 \%^{*}\right)$ and PDE-5 expression $\left(+170 \%^{*}\right)$ at day 5 .

\section{Conclusion}

An activation of the NO-cGMP pathway precedes resolution of acute anti-thy1 glomerulonephritis. Enhancing NO-cGMP signaling, for instance by using activators of soluble guanylate cyclase or inhibitors of PDE-5, may serve as pharmacological approach for regressing tissue fibrosis in established kidney disease. 